Ferrata Storti Foundation

\title{
Antithrombotic prophylaxis for surgery-associated venous thromboembolism risk in patients with inherited platelet disorders. The SPATA-DVT Study
}

Haematologica 2020

Volume 105(7):1948-1956

\section{Correspondence: \\ PAOLO GRESELE \\ paolo.gresele@unipg.it \\ Received: May 28, 2019. \\ Accepted: September 25, 2019. \\ Pre-published: September 26, 2019.}

doi:10.3324/haematol.2019.227876

Check the online version for the most updated information on this article, online supplements, and information on authorship \& disclosures: www. haematologica.org/content/105/7/1948

\section{(C)2020 Ferrata Storti Foundation}

Material published in Haematologica is covered by copyright. All rights are reserved to the Ferrata Storti Foundation. Use of published material is allowed under the following terms and conditions:

https://creativecommons.org/licenses/by-nc/4.0/legalcode. Copies of published material are allowed for personal or internal use. Sharing published material for non-commercial purposes is subject to the following conditions:

https://creativecommons.org/licenses/by-nc/4.0/legalcode, sect. 3. Reproducing and sharing published material for commercial purposes is not allowed without permission in writing from the publisher.
Francesco Paciullo, ${ }^{1}$ Loredana Bury, ${ }^{1}$ Patrizia Noris, ${ }^{2}$ Emanuela Falcinelli, ${ }^{1}$ Federica Melazzini, ${ }^{2}$ Sara Orsini, ${ }^{1}$ Carlo Zaninetti, ${ }^{2,3}$ Rezan Abdul-Kadir, ${ }^{4}$ Deborah Obeng-Tuudah, ${ }^{4}$ Paula G. Heller, ${ }^{5,6}$ Ana C. Glembotsky, ${ }^{5,6}$ Fabrizio Fabris, ${ }^{7}$ Jose Rivera, ${ }^{8}$ Maria Luisa Lozano, ${ }^{8}$ Nora Butta, ${ }^{9}$ Remi Favier, ${ }^{10}$ Ana Rosa Cid,,${ }^{11}$ Marc Fouassier, ${ }^{12}$ Gian Marco Podda, ${ }^{13}$ Cristina Santoro, ${ }^{14}$ Elvira Grandone,${ }^{15,16}$ Yvonne Henskens, ${ }^{17}$ Paquita Nurden, ${ }^{8}$ Barbara Zieger, ${ }^{19}$ Adam Cuker, ${ }^{20}$ Katrien Devreese, ${ }^{21}$ Alberto Tosetto, ${ }^{22}$ Erica De Candia, ${ }^{23,24}$ Arnaud Dupuis, ${ }^{25}$ Koji Miyazaki, ${ }^{26}$ Maha Othman ${ }^{27}$ and Paolo Gresele ${ }^{1}$

${ }^{1}$ Department of Medicine, Section of Internal and Cardiovascular Medicine, University of Perugia, Italy; ${ }^{2}$ Department of Internal Medicine, IRCCS Policlinico S. Matteo Foundation, University of Pavia, Pavia, Italy; ${ }^{3} \mathrm{PhD}$ program in Experimental Medicine, University of Pavia, Pavia, Italy; ${ }^{4}$ Haemophilia Centre and Haemostasis Unit, The Royal Free Foundation Hospital and University College London, London, UK; ${ }^{5} \mathrm{Hematología} \mathrm{Investigación,} \mathrm{Instituto}$ de Investigaciones Médicas Alfredo Lanari, Universidad de Buenos Aires, Buenos Aires, Argentina; ${ }^{6} \mathrm{CONICET}$, Universidad de Buenos Aires, Instituto de Investigaciones Médicas IDIM-, Buenos Aires, Argentina; ${ }^{7}$ Clinica Medica 1 - Medicina Interna CLOPD, Dipartimento Assistenziale Integrato di Medicina, Azienda-Ospedale Università di Padova, Dipartimento di Medicina, Università di Padova, Padova, Italy; ${ }^{8}$ Servicio de Hematología y Oncología Médica, Hospital Universitario Morales Meseguery Centro Regional de Hemodonación, IMIB-Arrixaca, Universidad de Murcia, Murcia, Spain; 'Unidad de Hematología, Hospital Universitario La Paz-IDIPaz, Madrid, Spain; ${ }^{10}$ Assistance Publique-Hôpitaux de Paris, Armand Trousseau Children's Hospital, French Reference Centre for Inherited Platelet Disorders, Paris, France; ${ }^{11}$ Unidad de Hemostasia y Trombosis, Hospital Universitario y Politecnico La Fe, Valencia, Spain; ${ }^{12}$ Consultations d'Hémostase - CRTH, CHU de Nantes, Nantes, France; ${ }^{13}$ Medicina III, ASST Santi Paolo e Carlo, Dipartimento di Scienze della Salute, Università degli Studi di Milano, Milan, Italy; ${ }^{14} \mathrm{Hematology}$, Department of Translational and Precision Medicine, La Sapienza University of Rome, Rome, Italy; ${ }^{15}$ Unità di Ricerca in Aterosclerosi e Trombosi, I.R.C.C.S. "Casa Sollievo della Sofferenza”, S. Giovanni Rotondo, Foggia, Italy; ${ }^{16} \mathrm{Ob} /$ Gyn Department of the First I.M. Sechenov Moscow State Medical University, Moscow, The Russian Federation; ${ }^{17}$ Hematological Laboratory, Maastricht University Medical Centre, Maastricht, the Netherlands; ${ }^{18}$ Reference Centre for Platelet Disorders, Bordeaux University Hospital Centre, Rythmology and Cardiac Modeling Institute (LIRYC), Xavier Arnozan Hospital, Pessac, France; ${ }^{19}$ Division of Pediatric Hematology and Oncology, Faculty of Medicine, Medical Center - University of Freiburg, Freiburg, Germany; ${ }^{20}$ Perelman School of Medicine, University of Pennsylvania, Philadelphia, PA, USA; ${ }^{21}$ Coagulation Laboratory, Department of Laboratory Medicine, Ghent University Hospital, Department of Diagnostic Sciences, Ghent University, Ghent, Belgium; ${ }^{22} \mathrm{Hematology}$ Department, S. Bortolo Hospital, Vicenza, Italy; ${ }^{23} \mathrm{Hemostasis}$ and Thrombosis Unit, Insitute of Internal Medicine, Policlinico Agostino Gemelli Foundation, IRCCS, Rome, Italy; ${ }^{24}$ Institute of Internal Medicine and Geriatrics, Università Cattolica del Sacro Cuore, Rome, Italy; ${ }^{25}$ Université de Strasbourg, Institut National de la Santé et de la Recherche Médicale, Etablissement Français du Sang Grand Est, Unité Mixte de Recherche-S 1255, Fédération de Médecine Translationnelle de Strasbourg, Strasbourg, France; ${ }^{26}$ Department of Transfusion and Cell Transplantation Kitasato University School of Medicine, Sagamihara, Japan and ${ }^{27}$ Department of Biomedical and Molecular Sciences, School of Medicine, Queen's University, Kingston, Ontario, Canada

\section{ABSTRACT}

M ajor surgery is associated with an increased risk of venous thromboembolism (VTE), thus the application of mechanical or pharmacologic prophylaxis is recommended. The incidence of VTE in patients with inherited platelet disorders (IPD) undergoing surgical procedures is unknown and no information on the current use and safety of 
thromboprophylaxis, particularly of low-molecular-weight-heparin in these patients is available. Here we explored the approach to thromboprophylaxis and thrombotic outcomes in IPD patients undergoing surgery at VTE-risk participating in the multicenter SPATA study. We evaluated 210 surgical procedures carried out in 155 patients with well-defined forms of IPD (VTE-risk: 31\% high, 28.6\% intermediate, $25.2 \%$ low, 15.2\% very low). The use of thromboprophylaxis was low (23.3\% of procedures), with higher prevalence in orthopedic and gynecological surgeries, and was related to VTE-risk. The most frequently employed thromboprophylaxis was mechanical and appeared to be effective, as no patients developed thrombosis, including patients belonging to the highest VTE-risk classes. Low-molecular-weight-heparin use was low (10.5\%) and it did not influence the incidence of post-surgical bleeding or of antihemorrhagic prohemostatic interventions use. Two thromboembolic events were registered, both occurring after high VTE-risk procedures in patients who did not receive thromboprophylaxis $(4.7 \%)$. Our findings suggest that VTE incidence is low in patients with IPD undergoing surgery at VTE-risk and that it is predicted by the Caprini score. Mechanical thromboprophylaxis may be of benefit in patients with IPD undergoing invasive procedures at VTE-risk and low-molecular-weightheparin should be considered for major surgery.

\section{Introduction}

Venous thromboembolism (VTE) is a severe and sometimes lethal complication of major surgery triggered by the release of pro-thrombotic substances from injured tissues, immobilization, medical comorbidities and favored by thrombophilia. It occurs in $20-25 \%$ of patients undergoing general surgery and in up to $60 \%$ of patients undergoing orthopedic surgery not receiving antithrombotic prophylaxis. $^{1-4}$

VTE can be largely prevented by the use of mechanical and/or pharmacologic antithrombotic prophylaxis. Mechanical thromboprophylaxis with compressive stockings or intermittent pneumatic compression devices reduces the risk of VTE by $64 \%$ and $60 \%$, respectively, while pharmacologic thromboprophylaxis with low molecular weight-heparin (LMWH) reduces VTE risk by $75 \%$, although it doubles the risk of major bleeding. ${ }^{4,7} \mathrm{~A}$ meta-analysis of clinical trials comparing mechanical versus pharmacologic thromboprophylaxis in general and orthopedic surgery found a $80 \%$ higher risk of deep vein thrombosis (DVT) (including asymptomatic and distal DVT) among patients treated with mechanical thromboprophylaxis but a $57 \%$ lower risk of major bleeding. ${ }^{8}$ Moreover, a systematic review comparing intermittent pneumatic compression with elastic compressive stockings in surgical patients found a prevalence of DVT of $2.9 \%$ in the first group and of $5.9 \%$ in the second. ${ }^{9}$ Recently, it has been observed that the addition of mechanical to pharmacologic thromboprophylaxis does not provide further benefit. ${ }^{10}$

The risk of VTE associated with surgery changes according to a series of variables. The American College of Chest Physicians (ACCP) evidence-based clinical practice guidelines classify surgical interventions into three VTErisk categories depending on the type of procedure. ${ }^{3}$ Moreover, individual VTE-risk can be estimated more accurately based on patient characteristics and risk factors using appropriate scores, one of the most widely used of which is the "Caprini score" which subdivides patients into four risk categories. ${ }^{11}$

The incidence of VTE in patients with inherited platelet disorders (IPD) undergoing surgical procedures at VTErisk is unknown, and no clinical trials or large case series have ever been reported, although several reports suggest that these patients may not be protected from thrombosis, ${ }^{12-15}$ especially when considering that some prophylactic antihemorrhagic treatments currently used in these patients for the preparation to surgery, like platelet transfusions or recombinant factor VII a (rFVIIa), ${ }^{16,17}$ increase VTE-risk. ${ }^{12,18,19}$

Moreover, no systematic studies on the use of thromboprophylaxis in patients with IPD undergoing surgery have been carried out, and no information on the safety of the prophylactic administration of LMWH to IPD patients is available, although isolated reports on the safe administration of anticoagulants to IPD patients have been published. ${ }^{20-24}$

Recently, the large retrospective, multicenter SPATA study evaluated bleeding complications and management of surgery in patients with IPD ${ }^{17}$ In the present study we evaluated the approach to thromboprophylaxis adopted for the IPD patients undergoing surgery at VTE-risk participating in the SPATA study. In particular, we aimed to assess current clinical decisions on VTE prevention, to estimate postoperative VTE-risk and to evaluate the association between the use of mechanical or pharmacologic thromboprophylaxis and clinical VTE incidence and surgical bleeding in IPD.

\section{Methods}

\section{Study population}

In the current sub-study we included all the surgical procedures performed in patients enrolled in the SPATA study according to well-defined laboratory and/or molecular genetic criteria ${ }^{17,25-27}$ for whom thromboprophylaxis should have been considered according to current guidelines, including major and minor invasive interventions. ${ }^{3,11,28}$ The decision to apply thromboprophylaxis was made by the attending physicians on an individual basis. Patients under 16 years of age were excluded due to the lower intrinsic VTE-risk in younger age..$^{29,30}$ Surgery definitions were previously reported. ${ }^{17}$ Given the significant in situ thrombotic risk of central venous catheter insertion interventions, ${ }^{31}$ these were also considered in the analysis as minor procedures with high local thrombotic risk.

A 48-item structured questionnaire on VTE-risk, thrombotic and bleeding events and antithrombotic prophylaxis had to be filled in for each at-risk procedure. The individual bleeding risk was estimated according to the type of IPD and previous individual bleeding history as assessed by the World Health Organisation (WHO)-bleeding score. ${ }^{17}$

The Institutional Review Board of the coordinating center 
approved this sub-study (CEAS Umbria, Italy, Approval $\mathrm{n}$. 13138/18).

For further details see the Online Supplementary Materials and Methods.

\section{Thromboembolic risk}

VTE-risk of surgical patients was estimated using the Caprini Score. ${ }^{32,33}$ The enrolled procedures were subdivided into four classes of risk depending on the Caprini score. Surgical procedures were also classified according to procedure-related VTE-risk in three groups as suggested by the 2008 ACCP. ${ }^{3}$ Both the Caprini and the procedure-related VTE-risk scores were centrally calculated based on the replies given by the participating investigators to the 48-item questionnaires. Further details are provided in the Online Supplementary Materials and Methods.

\section{Thrombotic outcomes}

Thrombotic outcomes were defined as any symptomatic thrombosis (deep venous, including distal, and superficial) and/or pulmonary embolism occurring within one month after surgery. For details see the Online Supplementary Materials and Methods.

\section{Bleeding outcomes}

Previous bleeding history was assessed using the WHO bleeding assessment scale (WHO-BS), ${ }^{34}$ while excessive bleeding occurring after surgery and the rate of success of emergency treatment of post-surgical bleeding were classified as previously described. ${ }^{17}$ Additionally, data about the need of blood transfusion after surgery were collected. Participating investigators were asked to provide informations about bleeding outcomes occurred both during and immediately after hospitalization for surgery.

The outcome of emergency treatment of excessive post-surgical bleeding was classified as successfully controlled, not responsive to treatment or re-bleeding. ${ }^{17}$

\section{Statistical analysis}

As this was a pilot, exploratory study without any a priori test hypothesis, we did not perform a formal sample size analysis. Variables not normally distributed were reported as medians and interquartile ranges (IOR), and differences were tested using the Mann-Whitney U test or the Kruskal-Wallis analysis of variance (ANOVA) test. Data are shown as medians and IOR. Categorical variables were analysed using the $\chi^{2}$ test. A Cochrane-Armitage test of trend was used to evaluate the correlation between dichotomous and ordinal variables. Logistic regression analysis was performed to identify predictors of excessive post-surgical bleeding, of heparin use, of the need for anti-hemorrhagic interventions and of the success of post-surgical bleeding management. All analyses were performed using SPSS version 22.0 (IBM Corporation, Armonk, NY, USA).

\section{Results}

\section{Patient characteristics}

Out of the 829 surgical procedures included in the SPATA study, 210 carried out in 133 patients met the inclusion criteria, 132 of which were performed in females $(63.8 \%)$, with 31 patients undergoing more than one procedure. Of these interventions, 110 (52.4\%) were carried out in 66 patients with 14 different forms of inherited disorder of platelet function (IPFD), and 100 (47.6\%) in 67 patients with seven different forms of inherited disorders of platelet number (IPND) (Online Supplementary Table S1). The median age at surgery was 45 years (IOR: 29-56; min
17 , max 88). Two patients $(0.9 \%)$, aged 19 and 26 years undergoing one procedure each, were heterozygous carriers of the FV Leiden mutation, although it should be considered that no systematic search for thrombophilic genetic mutations was made in the enrolled population; ${ }^{11}$ procedures $(5.2 \%)$ were performed in patients with a history of malignancy (median age 55 years; IQR: 52-79), and four $(1.9 \%)$ in patients with chronic obstructive pulmonary disease (COPD) (median age 51 years; IOR: 42-59). 65 interventions $(31 \%)$ were performed in patients with a Caprini score $\geq 5,60(28.6 \%)$ in patients with a score of 3 4, $53(25.2 \%)$ in patients with a score between 1 and 2, and $32(15.2 \%)$ in patients with a score of 0 . The median age was 32 years (IOR: 20-49) for patients with a score of 0,35 years (IOR: 27-46) for patients with a score of 1-2, 46 (IOR: 32-60) for patients with a score of 3-4, and 52 years (IOR: 41-61) for patients with a score $\geq 5$. Sixty-one interventions (29\%) (32 in patients with IPFD and 29 in patients with IPND) were low-risk, 114 (54\%) (55 in patients with IPFD and 59 in patients with IPND) were intermediate-risk, and 35 (17\%) (23 in patients with IPFD, 12 in patients with IPND) were high-risk. ${ }^{3}$ In low-risk procedures, the median age was 49 years (IOR: 33-58), in intermediate-risk 37 years (IOR: 28-53), and in high-risk 53 years (IOR: 33-62).

\section{Type of surgery and antithrombotic prophylaxis}

72 procedures were abdominal $(34.3 \%), 55$ gynecological $(26.2 \%), 41$ orthopedic (19.5\%), 14 urological $(6.7 \%)$, 10 cardiovascular $(4.8 \%)$, nine thoracic $(4.3 \%)$, six neurosurgical $(2.9 \%)$, and three spine surgeries (1.3\%). 90 interventions were major surgery (43\%) while the other 120 procedures $(57 \%)$ were minor invasive interventions followed by immobilization for $\geq 24$ hours. The oldest group of patients were those undergoing urological interventions (median age 58 years), while the youngest patients underwent gynecological surgery (median age 36 years). Malignancy was most frequent in patients undergoing thoracic surgery (Table 1). Of the overall 210 surgical procedures, $89 \%$ were elective and $11 \%$ urgent.

The Caprini score was higher in patients undergoing cardiovascular interventions and lower for abdominal interventions (Table 1).

Out of 210 surgical procedures, 49 (23.3\%) were managed with thromboprophylaxis; of these 27 (55.1\%) were managed with mechanical thromboprophylaxis alone, using either compression stockings ( 26 procedures) or intermittent pneumatic compression (one procedure), 19 (38.8\%) with LMWH alone, and three (6.1\%) with both methods (mechanical and pharmacologic).

Of the 49 interventions managed with thromboprophylaxis, 13 were orthopedic $(26.0 \%), 12$ gynecological $(24.5 \%)$, seven abdominal (14.3\%), seven thoracic $(14.3$ $\%)$, seven urological (14.3\%) and three neuro-spinal (6\%). LMWH prophylaxis was adopted in $22 \%$ of the orthopedic procedures, $12.7 \%$ of gynecological, $11 \%$ of thoracic, $11 \%$ of neuro-spinal surgery, $7.1 \%$ of urological and $4.2 \%$ of abdominal (Table 1 and Figure 1). The two patients carriers of factor $V$ Leiden mutation were both at intermediate VTE-risk and had a low WHO-BS (0 and 2, respectively). They both underwent gynecological surgery without thromboprophylaxis and did not develop VTE. Patients with a history of malignancy were all classified at an intermediate VTE-risk, and their median WHO-BS was 2. In these patients heparin was used in four procedures, 
Table 1. Inherited platelet disorders patient characteristics according to type of surgery.

\begin{tabular}{|c|c|c|c|c|c|c|c|}
\hline & $\begin{array}{l}\text { Abdominal } \\
\text { surgery } \\
\text { (N 72) }\end{array}$ & $\begin{array}{l}\text { Gynecological } \\
\text { surgery } \\
\text { (N 55) }\end{array}$ & $\begin{array}{l}\text { Orthopedic } \\
\text { surgery } \\
\text { (N 41) }\end{array}$ & $\begin{array}{l}\text { Urological } \\
\text { surgery } \\
\text { (N 14) }\end{array}$ & $\begin{array}{l}\text { Cardiovascular } \\
\text { surgery } \\
\text { (N 10) }\end{array}$ & $\begin{array}{l}\text { Thoracic } \\
\text { surgery } \\
\text { (N 9) }\end{array}$ & $\begin{array}{l}\text { Neuro/spinal } \\
\text { surgery } \\
\text { (N 9) }\end{array}$ \\
\hline Age in years, median (IQR) & $47(29-57)$ & $36(29-45)$ & $42(24-58)$ & $58(45-70)$ & $54(52-65)$ & $37(28-58)$ & $40(16-76)$ \\
\hline Females, N (\%) & $38(54.2)$ & $55(100)$ & $22(53.7)$ & $3(21.4)$ & $6(60)$ & $2(22)$ & $3(30)$ \\
\hline $\begin{array}{l}\text { Platelet count before surgery }\left(\times 10^{9} / \mathrm{L}\right) \text {, } \\
\text { median (IQR) }\end{array}$ & $\begin{array}{c}120 \\
(65-175)\end{array}$ & $\begin{array}{c}56 \\
(34-162.5)\end{array}$ & $\begin{array}{c}139 \\
(103-191.5)\end{array}$ & $\begin{array}{c}75 \\
(5-90)\end{array}$ & $\begin{array}{c}60 \\
(425-94)\end{array}$ & $\mathrm{NA}$ & NA \\
\hline Malignancy, N (\%) & $1(1.4)$ & $2(3.6)$ & $1(2.4)$ & $1(7.1)$ & $2(20)$ & $3(33)$ & $1(11)$ \\
\hline WHO bleeding score, median (IQR) & $2(1-4)$ & $2(1-2)$ & $2(1-3)$ & $2(1-3)$ & $3(1-3)$ & $2(1-3)$ & $3(2-4)$ \\
\hline Caprini score, median (IQR) & $2(1-4)$ & $3(2-5)$ & $4(2-7)$ & $3(2-4)$ & $7(3-8)$ & $2(1-5)$ & $2(0-8)$ \\
\hline Caprini class risk, median (IQR) & $1(1-2)$ & $2(1-3)$ & $2(1-2)$ & $2(1-2)$ & $4(3-4)$ & $2(1-4)$ & $2(1-4)$ \\
\hline Use of thromboprophylaxis, $N(\%)$ & $7(9)$ & $12(21.8)$ & $13(31.7)$ & $7(50)$ & 0 & $7(77)$ & $3(30)$ \\
\hline Mechanical thromboprophylaxis, N (\%) & *5 (6.9) & *7 (12.7) & $4(9.7)$ & $6(42.9)$ & 0 & $6(66)$ & $2(22)$ \\
\hline LMWH thromboprophylaxis, N (\%) & $3(4.2)$ & $7(12.7)$ & $9(22)$ & $1(7.1)$ & 0 & $1(11)$ & $1(11)$ \\
\hline Preoperative antihemorrhagic prophylaxis, $\mathrm{N}(\%)$ & $34(62.5)$ & $27(49.1)$ & $25(62)$ & $9(64.3)$ & $7(70)$ & $5(55)$ & $7(77)$ \\
\hline Any excessive post-surgical bleeding, N (\%) & $22(30)$ & $42(76.5)$ & $5(12.5)$ & $2(14.3)$ & $6(60)$ & $3(33)$ & $4(44)$ \\
\hline
\end{tabular}

IPD: inherited platelet disorders; IQR: interquartile range; LMWH: low molecular weight heparin; NA: not available; *: in some procedures both mechanical and LMWH thromboprophylaxis was employed.

mechanical thromboprophylaxis in five and no prophylaxis in two. No VTE was recorded in this population (Online Supplementary Table S2).

Of the procedures at high VTE-risk according to the Caprini risk stratification $(\mathrm{n}=65){ }^{33}$ thromboprophylaxis was adopted in $22(33.8 \%)$ (LWMH in 14, mechanical in six, and both in two) with no VTE events, while in 43 it was not adopted. Regarding procedures at intermediate VTE-risk ( $\mathrm{n}=60)$, thromboprophylaxis was used in 15 (25\%) (mechanical in 11 and pharmacologic in four), while of the procedures at low VTE-risk $(n=53)$ thromboprophylaxis was used in $10(18.9 \%)$ (nine mechanical, one both mechanical and pharmacologic), and of the procedures at very low VTE-risk $(n=32)$, thromboprophylaxis was used in only two patients $(6.2 \%)$ (one mechanical, one pharmacologic). According to the procedure-related VTE-risk stratification $^{3,35}$ high-risk procedures, 114 intermediaterisk and 61 low-risk, were performed. Thromboprophylaxis was adopted in $42 \%$ (nine pharmacologic and six mechanical) of the high-risk procedures, in $21 \%$ (six pharmacologic, 15 mechanical and three both) of the intermediate-risk and in $16.4 \%$ (four pharmacologic and six mechanical) of the low risk procedures. The choice of using LMWH, was significantly associated with the Caprini risk class $(P<0.001$ and $P=0.002$ respectively $)$ (Online Supplementary Table S3) and with the procedurerelated VTE-risk class $(P=0.007$ and $P=0.009$, respectively) (Figure 2A). The use of thromboprophylaxis with LMWH was similar between elective and urgent procedures: $10.2 \%$ versus $13 \%$ respectively ( $P=$ not significant [n.s.]).

Older age also independently predicted the use of pharmacologic thromboprophylaxis. In fact, LMWH-treated patients were significantly older (median age 67 vs. 42 years; $P<0.01)$ and had a higher median Caprini score $(8$ vs. 4; $P<0.01)$ than non-treated patients (Table 2). Additionally, history of cancer was more frequent in heparin users than in non-users (18\% vs. 3.2\%; P=0.018).

On the contrary, neither the WHO-BS nor sex distribution (both in IPFD and IPND) were significantly associated with LMWH use.

Mechanical prophylaxis was applied with graduated compression stockings in 30 procedures $(14 \%)$ and with intermittent pneumatic compression in one $(0.47 \%)$, while pharmacologic prophylaxis was undertaken with enoxaparin in 18 procedures (8\%), tinzaparin in one $(0.47 \%)$, dalteparin in one $(0.47 \%)$, and in two cases $(0.95 \%)$ type was not specified. Enoxaparin was administered at a median dose of 4,000 IU/day (IOR: 2,000-5,000 IU/day) for a median duration of 15 days (IOR: 7-18), starting on the day of surgery. The use of LMWH, as well as the use of any thromboprophylaxis, increased over time during the observation period covered by the study (LMWH: overall rate [OR] 2.5; 95\% confidence interval [CI]: 1.31-4.96; any thromboprophylaxis: OR 1.4; 95\% CI: 0.98-2.08) (Figure 3).

Thromboprophylaxis (pharmacologic and/or mechanical) was more common in patients with IPFD compared with those with IPND $(34.5 \%$ vs. $11 \% ; P<0.01)$ due to the greater use of mechanical thromboprophylaxis in the former $(24 \%$ vs. $3 \% ; P<0.01)$, even if there was no difference in VTE-risk between the two groups. LMWH was administered in $10 \%$ of procedures carried out in patients with IPND (10 procedures), and in 10.9\% of those carried out in patients with IPFD (12 procedures).

None of the patients affected by biallelic Bernard Soulier syndrome (bBSS) $(n=11)$ and Glanzmann thrombasthenia (GT) $(n=5)$ received pharmacologic thromboprophylaxis. This finding probably reflects the perception that the VTE-risk of these patients is low, as suggested by previous reports ${ }^{14}$ and the fear of bleeding. In IPND, LMWH was neither administered in patients with ACTN1-related thrombocytopenia $(n=5)$ nor in the only patient with $X$ linked thrombocytopenia (Online Supplementary Table S1). Median platelet count of the overall IPD population before surgery was $158 \times 10^{9} / \mathrm{L}$ (IOR: 120-287) in procedures followed by LMWH versus $120 \times 10^{9} / \mathrm{L}$ (IOR: 8-163) in those where $\mathrm{LMWH}$ was not administered $(P=$ n.s.).

\section{Thrombotic outcomes}

Two thromboembolic events were recorded $(0.95 \%$ of all interventions), both occurring in patients who did not receive thromboprophylaxis $(3.5 \%$ of non-prophylaxed 
Table 2. Differences between surgical procedures carried out with or without low molecular weight heparin thromboprophylaxis.

\begin{tabular}{|c|c|c|c|}
\hline & $\begin{array}{l}\text { LMWH use } \\
\qquad(\mathrm{N}=22)\end{array}$ & $\begin{array}{l}\text { LMWH non use } \\
\quad(\mathrm{N}=188)\end{array}$ & $\mathbf{P}$ \\
\hline Age, median (IQR) & $\begin{array}{c}67 \\
(79-55)\end{array}$ & $\begin{array}{c}42 \\
(25-54)\end{array}$ & 0.01 \\
\hline Females, N (\%) & $14(63.6)$ & $120(63.8)$ & n.s. \\
\hline $\begin{array}{l}\text { Platelet count before surgery } \times 10^{9} / \mathrm{L} \text {, } \\
\text { median (IQR) }\end{array}$ & $\begin{array}{c}158 \\
(120-287)\end{array}$ & $\begin{array}{c}120 \\
(8-163)\end{array}$ & n.s. \\
\hline IPFD, N (\%) & $12(54.5)$ & $98(52.1)$ & n.s. \\
\hline COPD, N (\%) & $2(9.1)$ & $2(1.1)$ & n.s. \\
\hline Malignancy, N (\%) & $4(18.4)$ & $7(3.2)$ & 0.018 \\
\hline $\begin{array}{l}\text { WHO bleeding score, } \\
\text { median (IQR) }\end{array}$ & $2(0.75-3)$ & $2(1-3)$ & n.s. \\
\hline Caprini score, median (IQR) & $8(5-12)$ & $4(2-6)$ & 0.02 \\
\hline Caprini class, median (IQR) & $4(3-4)$ & $3(2-4)$ & 0.01 \\
\hline Preoperative antihemorrhagic prophylaxis, N (\%) & $12(54.5)$ & $113(60.1)$ & n.s. \\
\hline Any excessive post-surgical bleeding, N (\%) & $4(18.2)$ & $46(25.8)$ & n.s. \\
\hline Treatment of post-surgical bleeding, N (\%) & $6(28.6)$ & $49(27.2)$ & n.s. \\
\hline Post-surgical bleeding duration, hours, median (IQR) & $6(4-8)$ & $6(1-6)$ & n.s. \\
\hline Failure of post-surgical bleeding control, N (\%) & $4(19)$ & $13(7)$ & n.s. \\
\hline Thrombosis, N (\%) & 0 & $2(1)$ & n.s. \\
\hline
\end{tabular}

IQR: interquartile range; COPD: chronic obstructive pulmonary disease; IPFD: inherited platelet function disorders; IPND: inherited platelet number disorders; LMWH: low molecular weight heparin; n.s.: not significant.

procedures). One was a pulmonary embolism (PE) in a bBSS patient who underwent mitral valve surgery, the other a femoral DVT in a GT patient occurring after the placement of a central venous femoral catheter for blood transfusions. Both patients were at high VTE-risk ${ }^{33}$ (Caprini score 12 and 8, respectively), had received prophylactic platelet transfusions before the invasive procedure, and had suffered excessive post-procedural bleeding prompting red blood cell transfusions. The patient suffering from PE was a 56-year-old obese woman affected by chronic obstructive pulmonary disease. She was then treated with therapeutic dose enoxaparin, but died in hospital from septic shock, disseminated intravascular coagulation and acute respiratory distress syndrome. The patient suffering from DVT was a 60-year-old woman and she was then treated with therapeutic dose enoxaparin for three months, without bleeding complications and with complete resolution of the femoral thrombosis. Both patients had previously undergone major elective surgery without thromboprophylaxis and without thrombotic complications. When dividing the included surgeries according to procedure-related VTE risk, in two of 35 high-risk procedures $(0.7 \%$, both IPFD) a VTE event occurred, while in 114 intermediate-risk procedures and 61 low-risk procedures no VTE occurred.

\section{Bleeding outcomes}

The percentage of patients who suffered from excessive bleeding after surgery was not significantly different in LMWH users compared with non-users (4 of $22: 18.2 \%$ vs. 46 of $188: 25.8 \% ; P=0.5)$ and no significant difference in bleeding duration after surgery was found between heparin users and non-users (Table 2). The rate of excessive bleeding was instead significantly higher in urgent $(45.5 \%)$ than in elective $(22.5 \%)$ procedures $(P<0.05)$.
Also the need of post-surgical blood transfusions did not differ between heparin users and non users $(18 \%$ vs. $19 \%$; $P=0.51)$ as well as the use of post-surgical anti-hemorrhagic interventions. In 57 cases emergency treatment of postsurgical bleeding was required $(27.1 \%)$, with platelet transfusions administered in 38 procedures, anti-fibrinolytic agents in nine, Desmopressin (DDAVP) in one, rFVII in one, other not specified treatment in six, and combination therapy with anti-fybrinolytic and DDAVP in two.

Thromboprophylaxis did not predict the need of post surgical anti-hemorrhagic intervention while the bleeding history did (Online Supplementary Table S4). Finally, heparin use was not significantly associated with the rate of success of emergency treatment of excessive post-surgical bleeding, although percentages of cases with treatment failures were numerically higher in LMWH users than in non-users (19\% vs. 7\%; OR 2.05, 95\% CI: 0.496-8.536; $P=0.321$ ) (Table 2 and Online Supplementary Table S5). Preoperatory prophylactic prohemostatic treatment was adopted in 125 procedures (59\%), in 78 with platelet transfusions, in nine with anti-fibrinolytic agents, in six with DDAVP, in three with activated rFVII and in three with a not-specified agent, in 12 with anti-fibrinolytic agents and DDAVP, in six with platelet transfusions, antifibrinolytic and DDAVP in combination, in four with platelet transfusions and anti-fibrinolytics in combination, in two with platelet transfusions and DDAVP in combination, in one with antifibrinolytic agents and a not-specified agent combination, in one with platelet transfusions and not-specified agent combination. Thromboprophylaxis with LMWH was adopted in 10 procedures $(11.8 \%)$ not managed with preoperatory prohemostatic prophylaxis and in $12(9.6 \%)$ of those managed with preoperatory thromboprohylaxis $(P=0.651)$. 


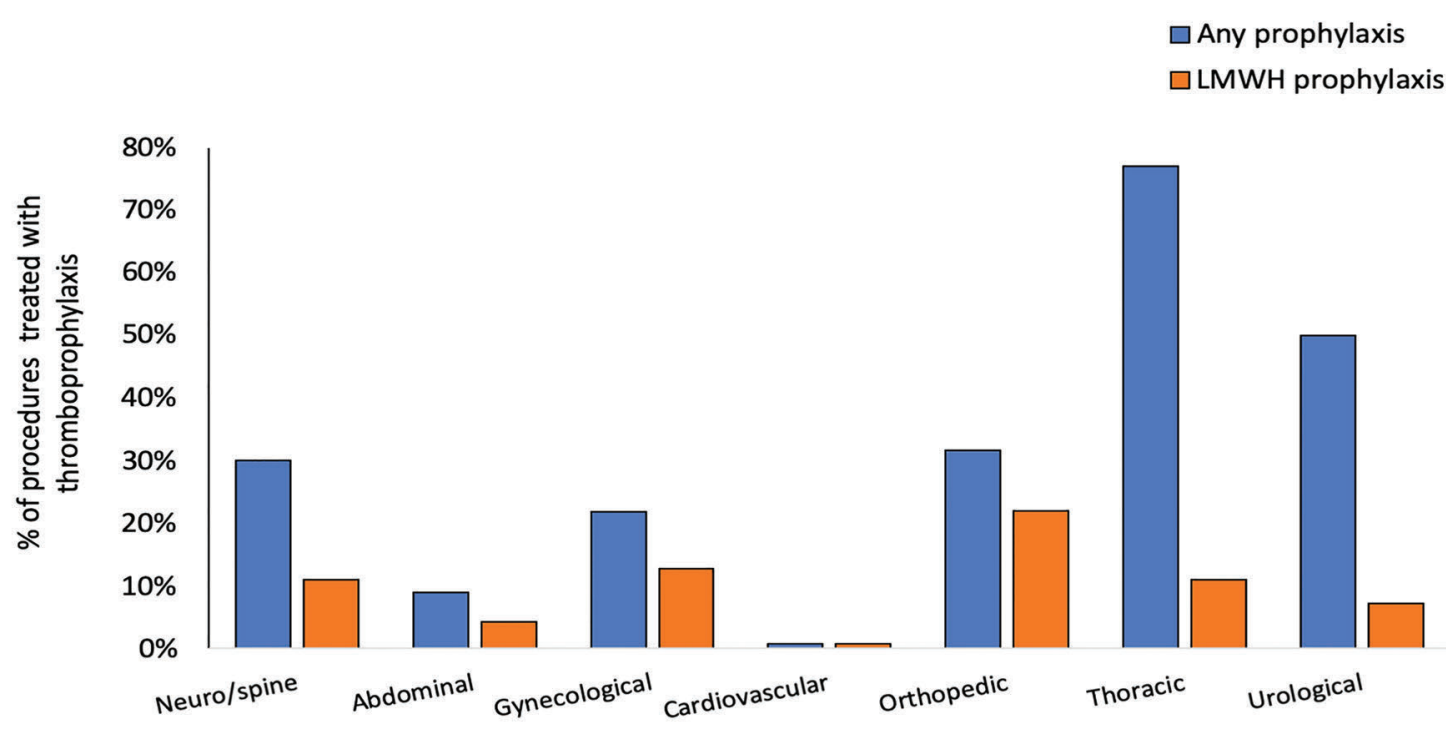

Type of surgery

Figure 1. Use of thromboprophylaxis in different types of surgery in the inherited platelet disorder population.

\section{Discussion}

Our data show that the current use of thromboprophylaxis in patients with IPD undergoing surgery at VTE-risk is low, probably due to fear of bleeding complications and to the belief that these patients are protected from VTE. In the general population the prevalence of pharmacologic thromboprophylaxis use has been estimated to be $17.7 \%$ in neurosurgery, $27 \%$ in abdominal surgery, $50 \%$ in gynecological surgery, $52 \%$ in cardiovascular surgery, $67 \%$ in urological surgery, $91 \%$ in orthopedic surgery, and $98 \%$ in thoracic surgery, ${ }^{35-38}$ while in our IPD population it was $0 \%$ in cardiovascular surgery, $9 \%$ in abdominal surgery, $11 \%$ in neuro and spinal surgery, $21.8 \%$ in gynecological surgery, $31.7 \%$ in orthopedic surgery, $50 \%$ in urological surgery, and $77 \%$ in thoracic surgery. In IPD patients, as expected, the most frequently employed thromboprophylaxis was mechanical, principally with elastic compression stockings. In otherwise healthy subjects undergoing general and orthopedic surgery the use of compression stockings was shown to exert a significant protective effect against VTE compared with no stockings ( $9 \%$ vs. $21 \%$; OR $0.35,95 \%$ CI: 0.28-0.43). ${ }^{39}$ In our IPD population this approach seemed to be effective, as no patients using post-surgery elastic compression stockings developed thrombosis, including patients at high risk based on the Caprini score.

In the general population, the risk of surgery-associated VTE in patients not undergoing thromboprophylaxis is strongly dependent on the Caprini score, with an incidence lower than $0.5 \%$ when the score is $0,3 \%$ when the score is $1-2,5 \%$ when the score is $3-4$, and $\geq 6 \%$ when the score is $\geq 5.11,3,40$ In our IPD population not receiving thromboprophylaxis, no VTE was observed in patients with a Caprini score $<5$ while in patients with a Caprini score $\geq 5$ symptomatic VTE occurred in $4.7 \%$ of the procedures. These data could suggest that the incidence of surgery-associated symptomatic VTE is indeed lower in patients with IPD that in healthy controls, at least when the Caprini score is not high. The ACCP guidelines classify surgical interventions in three groups depending on the risk of developing VTE: low risk $(<10 \%)$, including minor surgery and interventions not requiring patient immobilization, moderate risk (10$40 \%$ ), including gynecological and urological open surgery, and high risk (risk up to $80 \%$ ), including hip or knee arthroplasty, hip fracture surgery, spinal cord injury and procedures associated with high bleeding risk. ${ }^{3}$ In our IPD patients, in the high-risk group ${ }^{3} 58 \%$ of the procedures (21 interventions) were performed without prophylaxis and $9.5 \%$ of these were followed by VTE, while no VTE events were observed in moderate or low-risk procedures carried out without thromboprophylaxis. Of the two thromboembolic events recorded, one was observed in a GT patient undergoing a femoral vein catheter insertion and the other in a bBSS patient undergoing mitral valve surgery, both with a high individual VTE-risk (Caprini score of 8 and 12, respectively) and not receiving any thromboprophylaxis. Interestingly, the latter is, to our knowledge, the first case of VTE described in a bBSS patient. Pharmacologic thromboprophylaxis with LMWH was adopted in only $10 \%$ of all surgical procedures at VTE-risk in our IPD population. The use of thromboprophylaxis with LMWH increased over the observation period covered by the study, reflecting the increased awareness of the thrombotic risk of surgical procedures and of the efficacy of pharmacologic thromboprophylaxis. When heparin thromboprophylaxis was applied, its use did not seem to be guided by the assessment of the individual bleeding risk, but rather by the thromboembolic risk. Indeed, the Caprini score was strongly and independently associated with heparin use in our case series. No VTE was observed in patients undergoing LMWH prophylaxis, including in those belonging to the highest VTE-risk categories according to both the Caprini and procedure-related VTE scores. 
LMWH use was neither associated with an increased rate of excessive post-surgical bleeding nor with enhanced need for post-surgical antihemorrhagic intervention. Also the use of preoperative anti-hemorragic prophylaxis was similar in patients treated or not with LMWH. Thus, our results suggest that thromboprophylaxis with $\mathrm{LMWH}$ may be safer than anticipated in IPD patients. On the other hand, it should be pointed out that although LMWH did not significantly affect the success rate of emergency treatment of post-surgical bleeding, a numerically higher number of insuccess was observed in patients treated with LMWH. Thus, caution should be used when deciding about LMWH prophylaxis for IPD patients, especially for those at higher bleeding risk (e.g. more severe forms and/or patients with higher WHO bleeding scores). The use of post-surgical thromboprophylaxis with LMWH and the rate of VTE were similar between elective and urgent procedures, while the rate of excessive post-surgical bleeding was higher in urgent than in elective procedures, as expected.

Our study has several limitations. First, we only looked for symptomatic VTE, thus the incidence of total VTE may have been significantly underestimated due to the lack of a systematic instrumental diagnostics search of these events during post-surgical follow-up. Indeed, no calf or distal vein thrombosis was reported and the latter could have been overlooked, due to the low clinical expressivity and difficulty of diagnosis. However, the possible underestimation of distal DVT may not significantly diminish the clinical relevance of our observations because untreated distal DVT is associated with a low risk of proximal propagation and PE. ${ }^{32}$ Second, the retrospective nature of our study does not allow for definitive conclusions about the impact of heparin use on bleeding in patients with IPD. However, the collection of hemorrhagic post-surgical events was the main aim of our study and great emphasis was given to the careful evaluation of their occurrence. Moreover, the observational multicenter nature of our study, as already observed for other registries of populations with VTE, allowed us to gather a
A
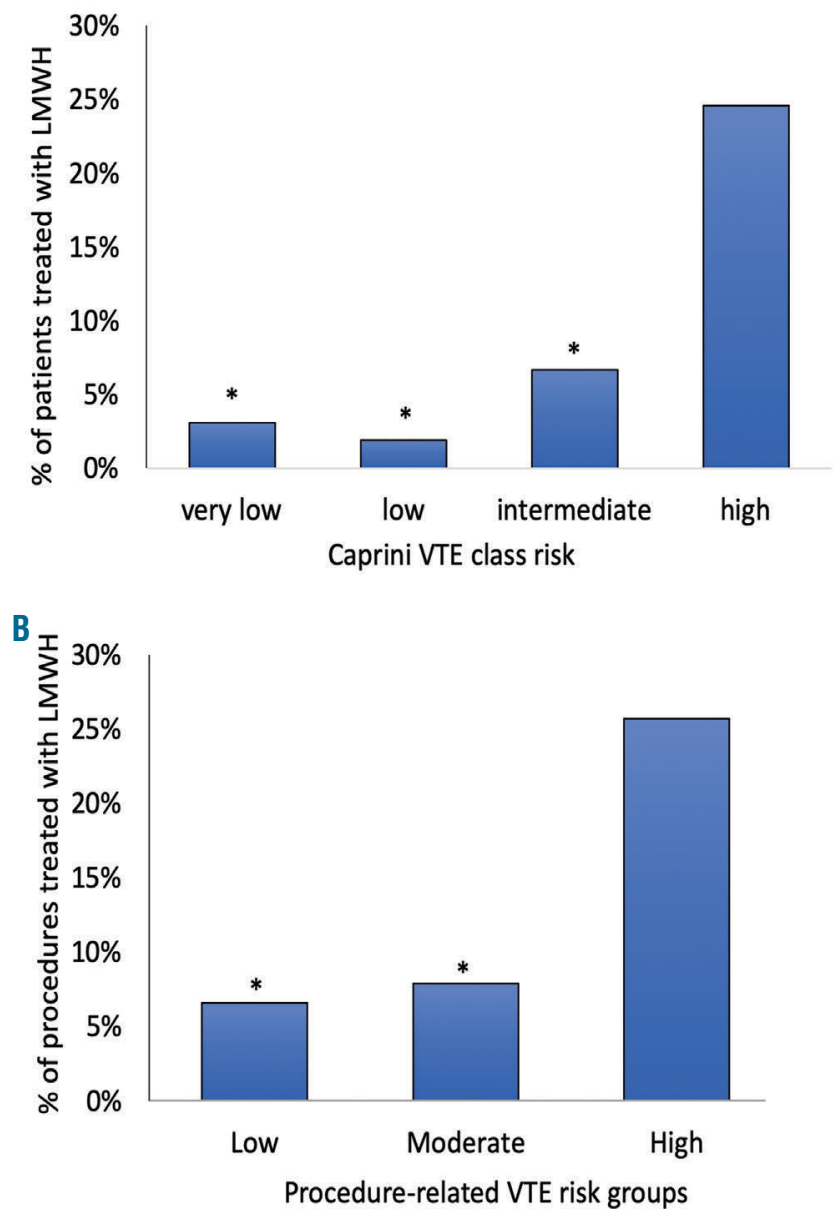

Figure 2. Use of low molecular weight heparin in inherited platelet disorde patients according to venous thromboembolism risk classes. Use of LMWH in IPD patients according to A) Caprini VTE class risk and B) procedure related VTErisk ( ${ }^{*} P<0.01$ vs. high-risk). LMWH: low molecular weight heparin; IPD: inherited platelet disorder; VTE: venous thromboembolism.

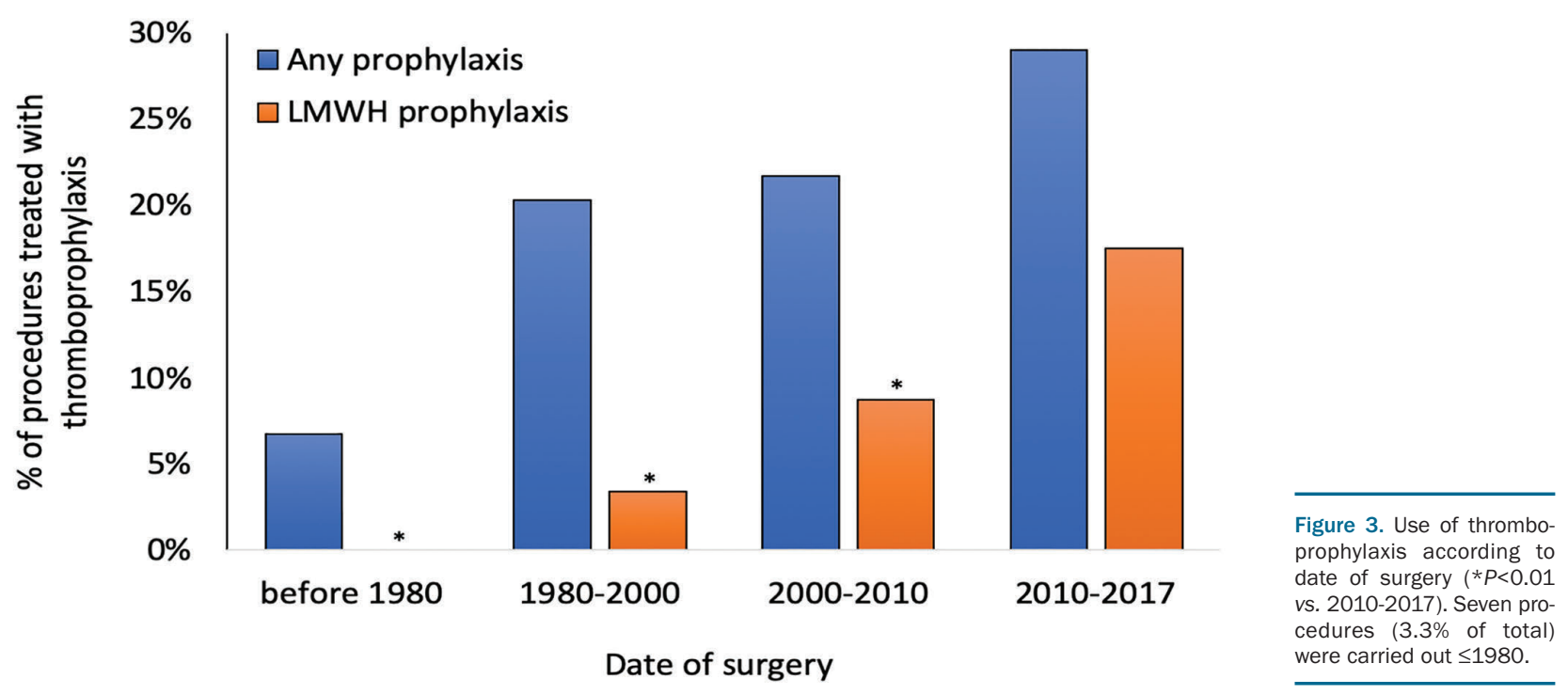


large patient series in an area difficult to explore with clinical trials, like subjects at high bleeding risk. ${ }^{41}$ Indeed, interventional clinical trials generally exclude patients at bleeding risk, limiting the generalizability of the evidence. Registries have been helpful for improving our understanding of the epidemiology, pattern of care and outcomes in such patient subgroups. ${ }^{41}$

Third, our study has a relatively small sample size and it involves a rather heterogeneous population undergoing a wide range of interventions performed over a fairly broad time period, thus limiting the strength of our results compared with studies carried out in the general population, especially when subgroup analyses are concerned. Although this is true, a case series of over 200 procedures carried out in rare-disease patients is not negligible in this clinical context, if one considers that phase 3 studies on LMWH prophylaxis in high-risk surgery and trauma have included between 100 and 440 patients. ${ }^{22-45}$

Despite the above limitations, to the best of our knowledge this is the first study which explored VTE-risk of surgical procedures in a large series of patients with IPD, and our results may represent the starting point for an evidence-based approach to the antithrombotic management of these subjects.

\section{Conclusions}

Our findings suggest that VTE incidence is low in patients with IPD undergoing at risk surgery. Moreover, among IPD subjects as well as in the general population, patients at high VTE-risk may be identified by the Caprini score. Our data also suggest that mechanical thromboprophylaxis may be of benefit in patients with IPD undergoing invasive procedures at VTE-risk and that LMWH should be considered for major surgery. Prospective studies are required to further clarify the impact of pharmacologic thromboprophylaxis on VTE and bleeding complications in patients with IPD undergoing surgery.

\section{Funding}

This study was promoted by the Scientific Working Group on Thrombocytopenias and Platelet Function Disorders of the European Hematology Association (EHA). LB and EF were supported by a scholarship grant from Fondazione Umberto Veronesi. This study was supported in part by a Telethon grant (GGP15063) to PG. NB holds grants from FIS-FONDOS FEDER (CP14/00024 and PI15/01457).

\section{Acknwoledgments}

The authors thank Dr. Giuseppe Guglielmini for help with statistical analysis, Prof. Marco Cattaneo (Università degli Studi di Milano, Italy), Prof. Christian Gachet (University of Strasbourg, France), Dr Alessandro Pecci, Drs Davide Rancitelli, Silvia Ferrari and Irene Bertozzi (University of Padua, Italy) and Dr. Giovanni Favuzzi (IRCCS Casa Sollievo della Sofferenza, San Giovanni Rotondo, Italy) for their contribution to the study.

We thank Dr. Rino Migliacci (Division of Internal Medicine, Ospedale della Valdichiana "S. Margherita", Cortona, Italy) and Prof. Carlo Balduini (Department of Internal Medicine, IRCCS Policlinico S. Matteo Foundation, University of Pavia, Italy) for the critical reading of the manuscript.i

\section{References}

1. Wheeler HB, Anderson FA, Jr. Prophylaxis against venous thromboembolism in surgical patients. Am J Surg. 1991;161(4):507-511.

2. Cohen AT, Tapson VF, Bergmann JF, et al. Venous thromboembolism risk and prophylaxis in the acute hospital care setting (ENDORSE study): a multinational crosssectional study. Lancet. 2008; 371(9610):387-394.

3. Geerts WH, Bergqvist D, Pineo GF, et al. Prevention of venous thromboembolism: American College of Chest Physicians Evidence-Based Clinical Practice Guidelines ( ${ }^{\text {th }}$ Edition). Chest. 2008;133(6 Suppl):381S453S.

4. Bozzato S, Galli L, Ageno W. Thromboprophylaxis in surgical and medical patients. Semin Respir Crit Care Med. 2012;33(2):163-175.

5. Agu O, Hamilton G, Baker D. Graduated compression stockings in the prevention of venous thromboembolism. Br J Surg. 1999;86(8):992-1004.

6. Urbankova J, Quiroz R, Kucher N, Goldhaber SZ. Intermittent pneumatic compression and deep vein thrombosis prevention. A meta-analysis in postoperative patients. Thromb Haemost. 2005; 94(6):1181-1185.

7. Mismetti P, Laporte S, Darmon JY, Buchmuller A, Decousus H. Meta-analysis of low molecular weight heparin in the pre- vention of venous thromboembolism in general surgery. Br J Surg. 2001;88(7):913930

8. Eppsteiner RW, Shin JJ, Johnson J, van Dam RM. Mechanical compression versus subcutaneous heparin therapy in postoperative and posttrauma patients: a systematic review and meta-analysis. World J Surg. 2010;34(1):10-19

9. Morris RJ, Woodcock JP. Intermittent pneumatic compression or graduated compression stockings for deep vein thrombosis prophylaxis? A systematic review of direct clinical comparisons. Ann Surg. 2010; 251(3):393-396.

10. Arabi YM, Al-Hameed F, Burns KEA, et al. Adjunctive intermittent pneumatic compression for venous thromboprophylaxis. N Engl J Med. 2019;380(14):1305-1315.

11. Gould MK, Garcia DA, Wren SM, et al. Prevention of VTE in nonorthopedic surgical patients: Antithrombotic Therapy and Prevention of Thrombosis, $9^{\text {th }}$ ed: American College of Chest Physicians Evidence-Based Clinical Practice Guidelines. Chest. 2012;141(2 Suppl): e227S-e277S.

12. d'Oiron R, Menart C, Trzeciak MC, et al. Use of recombinant factor VIIa in 3 patients with inherited type I Glanzmann's thrombasthenia undergoing invasive procedures. Thromb Haemost. 2000;83(5):644-647.

13. Varbella F, Bongioanni S, Gagnor A, et al. Primary angioplasty in a patient with the May-Hegglin anomaly, a rare heredity thrombocytopenia. A case report and review of the literature. Ital Heart J. 2005;6(4):214-217.

14. Girolami A, Sambado L, Bonamigo E, Vettore S, Lombardi AM. Occurrence of thrombosis in congenital thrombocytopenic disorders: a critical annotation of the literature. Blood Coagul Fibrinolysis. 2013;24(1):18-22

15. Nurden AT. Should studies on Glanzmann thrombasthenia not be telling us more about cardiovascular disease and other major illnesses? Blood Rev. 2017;31(5):287299

16. Gresele P, Falcinelli E, Bury L. Inherited platelet function disorders. Diagnostic approach and management. Hamostaseologie. 2016;36(4):265-278.

17. Orsini S, Noris P, Bury L, et al. Bleeding risk of surgery and its prevention in patients with inherited platelet disorders. Haematologica. 2017;102(7):1192-1203.

18. Aledort LM. Comparative thrombotic event incidence after infusion of recombinant factor VIIa versus factor VIII inhibitor bypass activity. J Thromb Haemost. 2004;2(10):1700-1708.

19. Schmidt AE, Henrichs KF, Kirkley SA Refaai MA, Blumberg N. Prophylactic preprocedure platelet transfusion is associated with increased risk of thrombosis and mortality. Am J Clin Pathol. 2017;149(1):87-94.

20. Humphries JE, Yirinec BA, Hess CE. Atherosclerosis and unstable angina in Bernard-Soulier syndrome. Am J Clin 
Pathol. 1992:97(5):652-655.

21. Gruel Y, Pacouret G, Bellucci S, Caen J. Severe proximal deep vein thrombosis in a Glanzmann thrombasthenia variant successfully treated with a low molecular weight heparin. Blood. 1997;90(2):888-890.

22. Shpilberg O, Rabi I, Schiller K, et al. Patients with Glanzmann thrombasthenia lacking platelet glycoprotein alpha(IIb)beta(3) (GPIIb/IIIa) and alpha(v)beta(3) receptors are not protected from atherosclerosis. Circulation. 2002;105 (9):1044-1048.

23. Seretny M, Senadheera N, Miller E, Keeling D. Pulmonary embolus in Glanzmann's thrombasthenia treated with warfarin. Haemophilia. 2008;14(5):1138-1139.

24. Girolami A, Vettore S, Vianello F, Berti de Marinis G, Fabris F. Myocardial infarction in two cousins heterozygous for ASN41HIS autosomal dominant variant of Bernard-Soulier syndrome. J Thromb Thrombolysis. 2012;34(4):513-517.

25. Noris P, Schlegel N, Klersy C, et al. Analysis of 339 pregnancies in 181 women with 13 different forms of inherited thrombocytopenia. Haematologica. 2014;99(8):13871394.

26. Civaschi E, Klersy C, Melazzini F, et al. Analysis of 65 pregnancies in 34 women with five different forms of inherited platelet function disorders. Br J Haematol. 2015;170(4):559-563.

27. Gresele P, Subcommittee on Platelet Physiology of the International Society on $\mathrm{T}$, Hemostasis. Diagnosis of inherited platelet function disorders: guidance from the SSC of the ISTH. J Thromb Haemost. 2015;13(2):314-322.

28. Poon MC, d'Oiron R, Zotz RB, et al. The international, prospective Glanzmann Thrombasthenia Registry: treatment and outcomes in surgical intervention. Haematologica. 2015;100(8):1038-1044.

29. Crous-Bou M, Harrington LB, Kabrhel C. Environmental and genetic risk factors associated with venous thromboembolism. Semin Thromb Hemost. 2016;42(8):808820.

30. National Institute for Health and Care Excellence. Venous thromboembolism in over 16s: reducing the risk of hospitalacquired deep vein thrombosis or pulmonary embolism. London, 2018. Available from: nice.org.uk/guidance/ng89.

31. Wall C, Moore J, Thachil J. Catheter-related thrombosis: A practical approach. J Intensive Care Soc. 2016:17(2):160-167.

32. Bates SM, Jaeschke R, Stevens SM, et al. Diagnosis of DVT: antithrombotic therapy and prevention of thrombosis, 9th ed: American College of Chest Physicians Evidence-Based Clinical Practice Guidelines. Chest. 2012;141(Suppl 2): e351S-e418S

33. Caprini JA, Arcelus JI, Hasty JH, Tamhane AC, Fabrega F. Clinical assessment of venous thromboembolic risk in surgical patients. Semin Thromb Hemost. 1991;17 Suppl 3:304-312.

34. Miller AB, Hoogstraten B, Staquet $M$, Winkler A. Reporting results of cancer treatment. Cancer. 1981:47(1):207-214.

35. Daley MJ, Ali S, Brown CV. Late venous thromboembolism prophylaxis after craniotomy in acute traumatic brain injury. Am Surg. 2015;81(2):207-211

36. Ho KM, Bham E, Pavey W. Incidence of venous thromboembolism and benefits and risks of thromboprophylaxis after cardiac surgery: a systematic review and metaanalysis. J Am Heart Assoc. 2015; 4(10): $\mathrm{e} 002652$

37. Amin AN, Lin J, Ryan A. Need to improve thromboprophylaxis across the continuum of care for surgical patients. Adv Ther 2010;27(2):81-93.

38. Dentali F, Malato A, Ageno W, et al. Incidence of venous thromboembolism in patients undergoing thoracotomy for lung cancer. J Thorac Cardiovasc Surg. 2008; 135(3):705-706

39. Sachdeva A, Dalton M, Lees T. Graduated compression stockings for prevention of deep vein thrombosis. Cochrane Database Syst Rev. 2018;11:CD001484.

40. Caprini JA. Thrombosis risk assessment as a guide to quality patient care. Dis Mon 2005;51(2-3):70-78.

41. Bikdeli B, Jimenez D, Hawkins $M$, et al Rationale, design and methodology of the computerized registry of patients with venous thromboembolism (RIETE). Thromb Haemost. 2018;118(1):214-224.

42. Marassi A, Balzano G, Mari G, et al Prevention of postoperative deep vein thrombosis in cancer patients. A randomized trial with low molecular weight heparin (CY 216). Int Surg. 1993;78(2):166170.

43. Bergqvist D, Benoni G, Bjorgell $O$, et al Low-molecular-weight heparin (enoxaparin) as prophylaxis against venous thromboembolism after total hip replacement. N Engl J Med. 1996;335(10):696-700

44. Kock HJ, Schmit-Neuerburg KP, Hanke J, Rudofsky G, Hirche H. Thromboprophylaxis with low-molecularweight heparin in outpatients with plastercast immobilisation of the leg. Lancet. 1995;346(8973):459-461.

45. Lassen MR, Borris LC, Nakov RL. Use of the low-molecular-weight heparin reviparin to prevent deep-vein thrombosis after leg injury requiring immobilization. $\mathrm{N}$ Engl J Med. 2002;347(10):726-730. 